Horizons philosophiques

\title{
La Nouvelle subjectivité et les technologies de l'information
}

\section{Monique Benoit et Jean Dragon}

Volume 11, numéro 2, printemps 2001

L’Europe, le laboratoire d'idées du XXI ${ }^{\mathrm{e}}$ siècle?

URI : https://id.erudit.org/iderudit/801186ar

DOI : https://doi.org/10.7202/801186ar

Aller au sommaire du numéro

Éditeur(s)

Collège Édouard-Montpetit

ISSN

1181-9227 (imprimé)

1920-2954 (numérique)

Découvrir la revue

Citer cet article

Benoit, M. \& Dragon, J. (2001). La Nouvelle subjectivité et les technologies de l'information. Horizons philosophiques, 11(2), 130-140.

https://doi.org/10.7202/801186ar d'utilisation que vous pouvez consulter en ligne.

https://apropos.erudit.org/fr/usagers/politique-dutilisation/ 


\section{LA NOUVELLE SUBJECTIVITÉ ET LES TECHNOLOGIES DE L'INFORMATION}

En insistant sur les effets de la culture de l'image renforcée par le développement des technologies de l'information et des communications (TIC)', le psychanalyste Serge Tisseron avance l'idée selon laquelle la multiplication des images a eu pour effet de leur faire perdre une partie de leur pouvoir de définition². La valeur accordée à l'image résiderait, selon l'auteur, moins dans la possibilité de se reconnaître à travers elle, ou encore dans sa signification, mais dans son caractère pragmatique et constructif. Les nouvelles technologies de l'information n'incitent-elles pas à les modifier sans cesse et à loisir pour des fins dites "utilitaires"? On ne se demanderait plus ce que l'image signifie et ce qu'elle évoque, mais ce qu'on peut en faire. Ainsi, le sens de la dimension picturale se situerait moins dans un procès référentiel que dans une praxis (souvent même auto-référentielle); comme si le "faire" s'était substitué au sens et que la possibilité de transformer l'objet prenait dorénavant le pas sur la conscience de soi. L'image se trouverait alors détachée de son contenu thématique et de son pouvoir d'évocation identitaire et intégrée dans un processus de transformation ludique qui fait du spectateur moins le témoin d'une histoire que d'une action dirigée contre l'intégrité de son contenu thématique et contre la charge mémorielle qu'elle supporte. Cette action sur l'image opérerait un détournement de sa fonction classique se soutenant de l'affirmation d'un droit de propriété non étranger à ce qui creuse l'opposition entre les cultures de l'«être" et de l'«avoir". Cette perception très "pragmatique", si ce n'est même transgressive associée à l'appropriation de l'image atteste également du renforcement de la liaison, toute "magique", entre la pensée et l'action, pire encore, de la subordination de la pensée à la main.

1. On définit généralement sous ce vocable l'ensemble des moyens de communication par câbles, par fibres optiques ou par satellites. Nous pensons notamment à l'informatique, aux moyens télévisuels et à la téléphonie que l'on compte actuellement parmi les plus grands du marché de l'information. Pour les besoins du présent texte, nous limitons notre propos aux moyens informatiques fournis par la toile de l'internet, lieu de prédilection des TIC.

2. Propos recueillis lors de sa communication intitulée «La Subjectivité par les objets» et présentée à l'Université du Québec à Montréal le 13 octobre 2000 au Colloque du CELAT : «Les Identités narratives : mémoire et perception". On peut aussi consulter quelques-uns de ses ouvrages : Le Mystère de la chambre claire : photographie et inconscient (Paris, Les Belles Lettres, 1996) et Le Bonheur dans l'image (Le Plessis-Robinson, France, Institut Synthelabo pour le progrès de la connaissance, 1996). 
Il est intéressant de noter la coalescence entre cette perte du pouvoir narratif de l'image et la réification d'une identité construite par le biais de sa plasticité (son jeu de substitution, d'altération, de greffe, etc.) et même de son morcellement (desapparatus et ipséitê). À cet effet, la toile de l'internet (comme pâle reflet des images tronquées de la toile picturale) constitue un espace de subjectivation impossible où la mémoire n'est sollicitée, le plus souvent, que comme "accident» et où une pensée magique, subordonnant la pensée à l'action, neutralise la réflexion et abolit la mémoire. L'hypothèse sur laquelle repose le présent texte vise à montrer combien internet et les TIC nous placent devant un «miroirelabyrinthe" qui ne réfléchit rien et où la subjectivation importe d'autant moins qu'avec l'«oubli», elle devient «impossible». À ce propos, il est intéressant de rapprocher l'oeuvre de deux auteurs, à savoir Georges Bataille et Jacques Lacan. Si la nouvelle subjectivité se manifeste non pas dans l'expression d'une opération parodique et souveraine telle que présentée chez Georges Bataille', elle repose toutefois sur l'absence d'une médiation à l'Autre comme on peut, en un sens, le comprendre chez Jacques Lacan. Comme cette médiation ne peut se faire en dehors de la langue 4 , la déconstitution du code symbolique, constant dans l'univers des internautes, fait en sorte que le sujet n'est plus en mesure de capter son «reflet». C'est là précisément, selon nous, que se joue la constitution négative d'une nouvelle subjectivité.

Si la culture visuelle occupe une large place sur la toile, l'organisation de l'information, quant à elle, n'a rien de "clairvoyant". Le regard s'ouvre sur sa propre cécité, une porte ouvre sur une autre porte, l'espace s'emboîte, s'uniformise sur la superposition de plans linéaires. En ce sens, la toile se présente comme le paradigme de cet esthétique où la virtualité nous fait "nier» en quelque sorte, non pas seulement la notion d'espace, mais la possibilité même d'inscription symbolique des limites du corps 5 . Est-il étonnant de réaliser que le Minotaure n'y est pas visible précisément parce que nous incarnons le monstre dans le labyrinthe, pire encore, nous coupons nous-mêmes le fil qui permettrait d'échapper à la superposition des illusions icariennes que propose la toile?

3. Chez Bataille, le sujet se capte toujours dans une opération de négation ludique et infinie. Ce qu'il capte de lui-même, c'est précisément ce qu'il met en jeu, de sorte que l'essence du sujet, très proche de Heidegger, se trouve dans l'ipséité. Voir, Jean DRAGON, «Pour une poétique bataillienne, la pratique de la dépossession», thèse de doctorat en sémiologie présentée à l'UQÀM, 2000, 525 feuillets.

4. Rappelons que Lacan, dans la foulée du structuralisme de la «Nouvelle critique" des années soixante, place la notion de symbolique comme une aporie constitutive de l'expérience de reconnaissance de soi. En fait, il va même plus loin que de parler de la toute-puissance du symbolique et pose l'expérience essentielle de soi dans le fantasme de son "contournement". 
Le présent texte se propose d'examiner, en trois séquences, certains enjeux des technologies de linformation et des communications (TIC) et leurs impacts dans la redéfinition de la subjectivité. Plus précisément, nous porterons notre attention sur la toile de l'internet et sur ses nombreux sites qui se veulent l'interface visuelle d'une nouvelle réalité de la quotidienneté, d'un réaménagement du signe et du signifiant, autant qu'un prolongement de la sphère académique 6 . Pour ce faire, nous exposerons d'abord quelques réflexions sur la structure et la forme même de la toile en tant que support actif (et non neutre) de l'information. Nous essayerons ensuite d'identifier certains effets de la mise en forme des informations dans le double horizon indissociable de l'expression et de la subjectivité. Nous étayerons enfin un regard critique nécessaire à l'égard de cette volonté de plus en plus généralisée d'intégrer internet aux méthodes d'enseignement, si ce n'est de redéfinir en partie l'enseignement lui-même à partir de ces nouvelles technologies.

\section{Doors with no house 7}

Si traditionnellement une porte délimitait un espace, ouvrait sur un autre territoire; la notion de "territoire" et même d' "espace" perdent leur sens. Nous avons alors une infinité d'avenues pour investir/délimiter un même territoire et même cent portes pour entrer dans la même pièce, dans la même garde-robe. Qu'il y ait cent portes, ou aucune, importe peu car nous sommes écartelés, disséminés même, dans le réseau d'une infinité de chemins et d'une multitude de seuils qui nous ramènent... à l'arrivée, à ce faux point de départ qui ressasse la

5. "Ces réaménagements nous obligent à faire le deuil du biocentrisme alors qu'on se relève à peine du deuil du géocentrisme et de l'anthropocentrisme". Voir, Louise POISSANT, «Écarts et monstruosité», dans Monstruosité, expression des passions, Montréal, L'instant même/Jaune-Fusain, 2000, p. 28. Voir également Monique BENOIT, aL'arraisonnement du corps des femmes et la puissance technicienne", conclusion de thèse de doctorat en sociologie présentée à l'UQÀM et intitulée "La stérilisation tubaire au Québec : une analyse des enjeux sociaux, médicaux et politiques", 2000, 346 feuillets.

6. La plupart des textes qui évoquent la nouvelle réalité virtuelle de l'internet l'abordent surtout à partir des TIC qui renvoient aux technologies de l'informatique et de la communication dont fait partie, bien entendu, la toile. Nous préférons plutót partir de la toile comme enjeu réel de la construction d'une nouvelle subjectivité. Dans le cadre du présent texte, la toile est vue comme horizon et nouveau paradigme de l'illustration de l'information et de l'uniformisation de la pensée par l'image, particulièrement dans le monde de l'éducation au Québec.

7. Tel le tableau de René Magritte intitulé Victoire (1939) représentant l'espace infini de l'horizon vu à travers une porte donnant sur le ciel, mais une porte qui ne tient pas à un mur; ouvrant ainsi sur l'horizon d'un ciel seulement limité par l'espace de la toile sur laquelle le peintre s'exprime. 
différence illusoire d'un même territoire. Nous assistons, en quelque sorte, à la chronique d'une présence incertaine qui désigne le spectacle de sa disparition sur un territoire non balisé à l'«image" d'une garde-robe où rien ne peut s'accrocher, ni être déposé. Un territoire aussi incertain pour l'habitus démontre à la fois une disproportion, si ce n'est d'une inversion, entre le contenant virtuel et le contenu parasité et illustre que la définition du corps propre s'effectue à même le morcellement et la dissémination en une multitude de points qui ne peuvent recomposer son «image plénière», lui assurer sa "propriété»8. Pire encore, puisque la virtualité suggère l'idée d'un corps sans limites qui ne repose sur rien, l'impossibilité de recomposer le corps où de façonner une identité propre semble corollaire d'un déni de mortalités. Michaël Lachance l'a déjà remarqué quand il écrit : "(...) la cyberculture produit un corps désincarné et monstrueux comme déni de la mortalité (...)»10. Ne sommes-nous pas dans un nouvel espace de perte qui permet de «ritualiser» une violence fondamentale et irreprésentable que la prolifération de l'image et le culte de la plastie expriment admirablement? Le desapparatus exprime moins la possibilité de se transformer que de se faire imposer une forme, moins la souveraineté qu'une aliénation. Une garde-robe sans porte convient parfaitement au mode d'inscription d'une subjectivité dont les contours flous sont l'expression d'une adaptation au monde virtuel. Cependant, le pouvoir de transformation ne procède pas fondamentalement de la mobilité plastique des individus, mais bel et bien d'une conscience de l'altérité. Certes, le pouvoir de transformation s'inscrit dans le plaisir d'un espace illimité. Ainsi, la garde-robe servira moins à se choisir un habit qu'à s'en débarrasser. Tel l'espace visuel et virtuelle occupé par la recomposition à "loisir» d'une disponibilité ludique à soi, la toile de l'internet prête à un exhibitionnisme de procuration, à une jouissance de la nudité, à l'extase radicale. Toutes ces formules inversées procèdent de la confusion généralisée à laquelle on se trouve convié. La mise en spectacle improvisée des fantasmes érotiques prolonge l'espace pornographique et l'inscrit dans un espace sans limites. Dans ce labyrinthe sans véritable espace, dans ces entrecroisements de galeries et de couloirs virtuels, l'anonymat procure aux internautes la possibilité de

8. Peut-on entrevoir dans le "vécu en réseaun un amalgame monstrueux où la toile se compose précisément de la dissémination de soi? De plus, si le sujet s'appropriait limage par le pouvoir de la praxis, il en est aussi réduit à être sujet des effets de cette opération qui le subordonne à un objet dont l'existence est liée aux regards étrangers et anonymes du réseau.

9. Ne sommes-nous pas habitués à entrevoir dans la toile un adanger mortel», ici, celui de vivre en périphérie de soi?

10. Michaël LACHANCE, "La Monstrueuse normalité», dans Monstruosité (...), p. 58. 
jouir d'eux-mêmes en exposant ce qu'ils réservent habituellement à la sphère du privé. Les cent portes de la garde-robe sont l'interface d'une redéfinition des territoires certes de lidentité mais aussi de l'«intime". La place publique ne se rencontre que sur un monde soliloque qui atténue la frontière avec l'intimité, de sorte que la "place publique" s'y démarque surtout par l'exhibition des écarts qui s'y affichent et qui donnent parfois l'impression de se retrouver en une sorte de nouveau cabinet des "monstruosités" et où la toile fait figure de "foire médiévale»!

Nous assistons à une complète "horizontalité» sans référence au moindre point d'ancrage; la "verticalité» étant comprise comme étant l'expression d'un pouvoir critique. En fait, nous substituons le deuil d'une certaine transcendance dans la création d'un sentiment de "toutepuissance" où nous ne savons pas trop, face à l'horizontalité de ces technologies, clairement départager la part de fascination de l'expression de nos besoins véritables et surtout humains. Enfin, à l'impossibilité d'une dialectique de subjectivation s'ajoute une transgression simulacrale qui invalide le caractère immanent d'une certaine sacralité de l'expérience ${ }^{11}$.

\section{Le travail du «parlêtre»}

Le travail d'une certaine langue non étrangère à la culture de la toile tend également à faire l'économie du passage à l'autre de sorte que l'expérience de reconnaissance dialectique de soi achoppe puisqu'elle se produit en dehors des conditions qui la rendent possible. Nous remarquons cette pratique de la langue, toute étrangère aux normes grammaticales et à l'orthographe, particulièrement chez les chateurs ${ }^{12}$. Nous assistons alors moins à l'expression de "soì qu'à un certain anonymat qui tient lieu de soi et d'un "non-soi» qui fait office de l'autre. De façon générale, les modalités de communication orientent alors moins l'expérience du locuteur vers la "communauté" qu'elles attestent de la faillite chez lui d'une tentative incessante de se faire reconnaître sous le registre d'un certain "écart" (de soi et de la communauté) et d'une certaine plastie entendue comme saturation du sens, hybridité de la langue et plus particulièrement ici de l'information à partager. Dans cette économie du «ratage» et même du "dérapage», nous assistons à l'exposition d'une "pensée magique» où l'on a tendance à croire en l'autonomie du verbe et en une polysémie du mot inféodé à une grammaire conçue

11. La transgression est toujours conditionnée par un interdit localisé qualitativement et temporellement. Qu'advient-il si son renversement, voire son dépassement ne porte plus sur sa nature et sa possibilité d'inscription «historiale"? Un pur espace simulacrale privé de son pouvoir de transformation? 
comme un «moteur de recherche" (fureteur) ou même comme un générateur de textes (et même d'hypertextes)! Hughes Marchal faisait remarquer ${ }^{13}$ que le principe des "Cent mille milliards de poèmes" de Raymond Queneau où l'auteur n'est plus scripteur, mais concepteur d'algorithmes textuels représentait une belle anticipation de cette grammaire textuelle sans aucun rapport avec la subjectivité et même tout cadre référentiel. Peut-on alors encore parler d'un rapport entre le lecteur et l'auteur?

Cette expérience d'une langue, en quelque sorte, "totémique" supportant une pensée «indifférente" à la différence, qui résulte sans doute de son appauvrissement, parvient difficilement à masquer un imaginaire qui propose le fantasme de (se) saisir en dehors de toute fonction symbolique. Faut-il rêver alors à l'inexistence d'une langue («totale» parce que réduite à la rumeur) qui correspondrait à de telles visées, une langue adaptée à cette "passe" où la volonté de reconnaissance tient lieu d'un au-delà/en-deçà du symbolique? Kossé? Le tout y figure sous forme de substantivation pas même du contenu, ni même très exactement de la forme, tout juste d'une résonance encline à assurer le travail des associations cognitives. Le rêve quoi! Rêve d'une certaine indolence où l'on serait pris en charge par nos fantasmes, où l'on serait "parlé", que disons-nous, "agi» et où nous serions convoqués à la foire du réel sous le mode d'une douce "absence» indolente! Nous assistons non pas au fantasme classique d'auto-engendrement tel que certains s'emploient à le reconnaître dans les visées spéculaires de la tradition moderne, mais bien à l'autarcie du non-moi qui s'enfante indéfiniment et dont la quiddité réside davantage dans sa "plasticité" que dans la filiation entre le non-moi et ses épigones. L'autarcie du non-moi ne peut conduire qu'à l'amplification d'un narcissisme fondamental et même à lillusion d'une autarcie référentielle où tout se vaut et se tient dans le registre de l'expression; où tout se justifie et surtout où le monde, tout comme la connaissance, se révèlent des constructions démiurgiques ${ }^{14}$.

12. Le chat est particulièrement populaire actuellement chez les adolescents et adolescentes précisément à cause de l'usage disons très alibre» de la langue. On y réinvente parfois les mots à partir de définitions qui appartiennent à une communication restreinte ayant pour but, le plus souvent, d'exclure par le fait mème les autres.

13. Voir "Le Texte au risque du virtuel : $\theta$-littérature et écrits d'écrans", dans Magazine littéraire, no 392 (novembre 2000), p. 34-36.

14. Que dire de l'hypertexte sans début et sans fin, lequel s'édifie à loisir au moment qui coincide avec la volonté du lecteur, ce texte dorénavant privé d'origine et d'incipit? Un événement qui renforce le ludisme et le narcissisme? 


\section{La transmission du savoir par "TIC"?}

(...) en prétendant inscrire le monstrueux dans la sphère du pédagogique et de la bonne conscience - il faut successivement apprivoiser le monstre, l'accepter, le banaliser et enfin ne plus le voir (... $)^{15}$

On sait que le développement des moyens de communication pousse beaucoup de professeurs à réviser leurs méthodes d'enseignement, y voyant sans doute une occasion de réaliser plus efficacement leurs objectifs pédagogiques et surtout de mieux "rencontrer" le profil de leurs "apprenants". Bien entendu, ces moyens sont intégrés avec plus ou moins de bonheur selon qu'ils se prêtent ou non aux disciplines et selon les habiletés ou même le désir qu'ont certains enseignants de les inscrire en classe. Dans ces domaines, il est clair, par exemple, que l'enseignement à distance (EAD) subit d'énormes pressions face aux technologies de l'information et de la communication (TIC). Les TIC obligent de plus en plus I'EAD à oeuvrer sur un "marché» externe au monde habituel de la pédagogie (écoles, collèges ou même universités) et à entrer sur la toile. Le problème surgit lorsqu'on intègre ces technologies par effet de fascination et qu'on en suggère, voire on en impose le recours moins par souci de formation que par rigueur administrative. Tout ceci est bien connu et les enjeux sont d'une clarté affolante. L'accroissement de l'"école en réseau" et de l'intégration des nouvelles technologies dans l'enseignement correspond souvent moins à la vocation de servir la "clientèle" à distance qu'à cette "rentabilisation maximale" des fonds publics qui visent l'augmentation non pas de la qualité de la formation mais de la "performance de diplomation". Ceci n'est pas étranger d'ailleurs au discours d'excellence ou du moins ce que l'on qualifie d'excellent actuellement dans les sphères de l'éducation. À quand alors, sur le modèle de nos voisins américains, la bonification salariale pour un haut taux de diplomation? On assiste actuellement à la mise sous "surveillance» des institutions académiques dont le financement dépend de plus en plus de leur soumission à la rentabilité conditionnelle au "contrat de performances 16 .

La tentation est grande de croire que les nouvelles technologies et particulièrement "l'école en ligne" ouvriront les "marchés" et permettront de meilleures performances financières des institutions. Non seulement il en va de la qualité des formations dispensées et des condi-

15. Jean-Pierre VIDAL, "Le Regard évidé», dans Monstruosité (...), p. 36.

16. Les administrations seront-elles désormais soumises à une concurrence fondée sur un «pointage à la performance»? Si tel est le cas, comment éviter que ce processus de classification des Universités mette en péril la diversité de leurs objectifs et la reconnaissance de leurs singularités? 
tions salariales du corps enseignant, mais aussi d'une illusion où les petites institutions risquent fortement non pas d'attirer de la clientèle, mais d'en perdre et ainsi de faire en sorte que la clientèle soit toute aussi virtuelle que la qualité de la diplomation... Les enjeux de la formation en ligne sont tellement explicitement financiers que les administrations adoptent parfois des attitudes qui n'hésitent pas à fragiliser l'enseignement traditionnel. Sans compter également que la question d'un contenu culturel distinct devient plus que problématique dans la situation d'une "ouverture des marchés". Ce dont on parle moins dans le développement de ce "savoir virtuel", c'est de l'effet même de la forme de la toile sur les profils d'apprentissage, de sa capacité de transmission d'un «héritage culturel", pire encore, on ne prend pas même en charge une réflexion fondamentale et critique sur les bouleversements ontologiques qui président à ce que l'on pourrait appeler l'émergence de cette "nouvelle subjectivité» avec laquelle nous devons composer.

Nous nageons en plein "pédagogisme" où la connaissance y est moins l'objet d'un apprentissage que d'une construction. Comment éviter alors la confusion entre savoir et information où l'on prend l'information pour le savoir? En effet, le contenu y est souvent moins important en ce que le contenant et le "processus d'apprentissage" (où l'on "apprend à apprendre" en quelque sorte) semble prendre le pas sur un ancrage à la transmission et où il importe moins, en définitive, de savoir mais d'être en mesure de démontrer que l'on sait. Ceci n'est pas étranger à la domination qu'exerce, depuis plus de vingt ans, la psychologie sur les théories contemporaines de l'éducation, lesquelles sont devenues le fondement culturel de l'enseignement ${ }^{17}$. II faut donc prévoir que l'introduction des TIC dans le domaine de l'éducation ne recèle pas que des avantages, ne serait-ce que financiers pour les institutions. Leur usage en remplacement de l'effort académique soutenu déployé par l'enseignant peut avoir des effets désastreux pour le mandat culturel des institutions. Les TIC contribueront très certainement à faire participer davantage la culture d'une rationalisation opératoire obtuse non étrangère à l'irresponsabilité d'une logique étroitement comptable. Notons que le vocabulaire de la réussite qui les accompagne y est calqué sur celui de l'entreprise: «'école comme marché", les "contenus comme objets de transactions", la "plus-value" de la formation en ligne, la "gestion de classe" (ce qui va de paire avec ce socio-constructivisme derrière l'«apprentissage par compétences), le "services en ligne» et même les "contrats de performance». Sur le plan institutionnel, l'effet d'urgence/fascination se fait nettement sentir de la

17. Voir à ce sujet Gilles GAGNÉ, «Introduction» à Main basse sur l'éducation, Québec, Nota Bene, 1999. 
part des administrations désireuses que l'on effectue le plus rapidement possible des changements législatifs favorisant une reconnaissance plus large des diplômes acquis par les TIC, d'autant plus que le «marché» est grandement lorgné par le privé face auquel on accuse déjà un certain retard. Ainsi, si la "présence des tic" devient de plus en plus la voie d'une "réussite" ces dernières années, la pression risque d'être encore plus forte alors que l'on donne des assises toutes virtuelles au désir d'une école entrevue comme lieu d'une "promotion sociale".

Autant de glissements de sens mettent en lumière le dérapage éducatif auquel on est convié. Le "projet éducatif" mis en place à l'aide des TIC a certainement pour but d'atteindre l'équilibre budgétaire en éducation; mais qu'en est-il de la réussite éducative pour les jeunes? Qui peut actuellement démontrer le lien entre les TIC et l'acquisition de connaissances par le biais de la formation? En faisant ainsi rimer la réussite scolaire avec la satisfaction face aux nouvelles formes d'apprentissages (sinon qu'à des apprentissages satisfaisants), les professeurs qui enseignent par-TIC s'exposent à des problèmes quand précisément ils semblent se satisfaire d'une réussite éducative fondée sur la satisfaction des apprentissages, même si celle-ci n'entraîne pas une véritable motivation pour les cours. En claire, l'élève n'aura plus à éprouver une quelconque motivation pour les cours, il n'aura qu'à avoir envie de s'amuser ${ }^{18}$. Or, il faut évidemment plus pour connaître. On semble confondre alors la volonté de réussir au divertissement et l'acquisition de connaissances issues de l'effort intellectuel à une satisfaction immédiate. Si l'on a affaire uniquement à du ludique que fera-t-on de la connaissance à transmettre d'une génération à l'autre? Dans ce passage du monde de l'activité intellectuelle à une forme de dilettantisme engendrée par les TIC, on semble également associer la motivation à la compétence; laissant ainsi croire que le savoir se résume à l'information et la transmission de connaissances à la communication de données. Si seulement ces nouvelles formes de transmission de linformation par les TIC, non confondues avec l'univers de la connaissance par l'éducation, donnaient lieu à un espace ludique recomposant les individus en être libres! Or, il n'en est rien puisque la confusion entre savoir et information, issue de l'assujettissement à la technologie, mène, et c'est bien connu, à une forme d'aliénation des êtres peu propice à l'imagination et la créativité.

18. Comment interpréter le taux d'échec alarmant des jeunes étudiants en informatique qui semblent parfois croire au prolongement, dans l'univers académique, de cette osmose ludique qu'ils entretiennent avec leur écran cathodique? Que dire des effets de ces échecs élevés qui rejaillissent sur l'ensemble des disciplines fondamentales? 
Dans un tel contexte où l'on suppose une "autonomie» préalable à l'apprentissage chez l'étudiant on peut facilement concevoir le travail scolaire, celui-là étant organisé dans un contexte para-scolaire, comme une pure fiction. De même, les exigences demandées à l'enseignant sont exprimées dans l'affranchissement de ses obligations académiques et se mettent en place essentiellement en terme de formulation d'un «menu à la carte». On aura remarqué que les exigences sont dès lors demandées à l'enseignant alors que l'autonomie est offerte à l'étudiant... Ici les cours y semblent moins destinés à favoriser l'accès à une véritable autonomie intellectuelle par la rencontre face à face, en magistral, avec le, ou la, professeur qu'à ne pas entraver le confort de celle qui lui est préalable, c'est-à-dire le loisir de choisir. Aussi l'autonomie présupposée aux "apprenants" semble a priori se fonder sur le pari de la distance bien que ce soit surtout là la vocation première de l'EAD. Cette autonomie repose également davantage sur leur désir de promotion que sur l'acquisition d'habiletés réelles et moins encore sur l'accès à une connaissance véritable.

Enfin, pourra-t-on se réjouir de ce que les TIC rencontrent d'ailleurs beaucoup d'obstacles dans la ufidélisation" d'une "clientèle" portée au "zapping". Son souci premier - hormis l'élaboration de contenus, l'amélioration des forums de discussion, la création des "boîtes de dialogues", la disposition des "banques de données" - consiste à favoriser la création "d'une proximité en ligne»19? Non seulement l'espace n'est-il plus posé comme une donnée problématique, mais son absence devient le lieu d'élaboration d'un fantasme en réseau; celui d'une communauté d'appartenance toute virtuelle qui n'a rien à envier, par exemple, à la communauté négative dont nous entretenait Maurice Blanchot il y a de cela 20 ans. Si l'sin-existence" de la communauté chez l'auteur français se tissait d'un travail de "subjectivation" possible par une oeuvre qui nous positionnait, bien que négativement face au "réel», peut-on en dire autant de l'existence amorphe (pour ne pas dire apathique) que nous propose une dissémination de soi dans l'anonymat du réseau? Peut-on, encore une fois, s'étonner que cet espace virtuel offre bien peu à une dialectique de subjectivation? En effet, comment une conception de l'image privée de son pouvoir narratif et une conception de la "lettre" saisie en dehors de sa fonction symbolique peut-elle fonder la

19. «L'enseignement en ligne vous réserve une 'place'»!... Peut-on lire parfois sur des réclames publicitaires! Étrange! Que dire du statut d'étudiant virtuel à qui on assure une place... dans la file... Cette situation très anonyme - qui devrait marquer un certain confort psychologique pour le candidat ne manque pas d'évoquer un certain folklore - semble d'autant plus ironique que cette place assurée reste, somme toute, «virtuelle». 
possibilité de la reconnaissance de soi? Ne peut-on pas voir précisément dans cette inversion du sens même de ces domaines respectifs le fond du problème? En accentuant le caractère sensible de la lettre dans l'ingénierie du texte, n'a-t-on pas fait du texte quelque chose qui signifie moins qu'il n'apparaît; laissant ainsi ouvert le champ esthétique et sensible à l'hypertrophie d'une culture visuelle axée sur l'appropriation de tout contenu référentiel et thématique. Ce n'est plus le signifiant qui prend d'assaut le signifié, mais bien la dislocation du signifié qui fait office de signifiant. En plus de ruiner la possibilité d'un rapport positif entre la lecture et le lecteur, une telle conception de la langue et du texte fait de la "chose littéraire" une icône qui apparaît et dont l'intérêt principal réside dans le plaisir des yeux. Un tel "degré zéro" de la lecture renvoie davantage à une esthétique strictement visuelle qu'à une conception du texte vouée à la connaissance fondamentale et au savoir.

\author{
Monique Benoit \\ Département d'anthropologie \\ et de sociologie \\ Université Laurentienne de Sudbury, \\ Ontario
}

\author{
Jean Dragon \\ Département de philosophie \\ Collège Édouard-Montpetit, \\ Membre du Centre d'Études en Lettres, \\ Arts et Traditions (UQÀM)
}

